\title{
Politique
}

Politique

\section{Lizette Jalbert et Lucille Beaudry, Les métamorphoses de la pensée libérale : sur le néo-libéralisme actuel, Montréal, Les Presses de l'Université du Québec, collection « Études d'économie politique », 1987, 240 p.}

\section{Max Nemni}

Numéro 14, automne 1988

Sport et politique et Le NPD

URI : https://id.erudit.org/iderudit/040608ar

DOI : https://doi.org/10.7202/040608ar

Aller au sommaire du numéro

\section{Éditeur(s)}

Société québécoise de science politique

ISSN

0711-608X (imprimé)

1918-6584 (numérique)

Découvrir la revue

Citer ce compte rendu

Nemni, M. (1988). Compte rendu de [Lizette Jalbert et Lucille Beaudry, Les métamorphoses de la pensée libérale : sur le néo-libéralisme actuel, Montréal, Les Presses de l'Université du Québec, collection «Études d'économie politique ", 1987, 240 p.] Politique, (14), 179-184.

https://doi.org/10.7202/040608ar d'utilisation que vous pouvez consulter en ligne. 
Lizette Jalbert et Lucille Beaudry, Les métamorphoses de la pensée libérale: sur le néo-libéralisme actuel, Montréal, Les Presses de l'Université du Québec, collection "Études d'économie politique", $1987,240 \mathrm{p}$.

De nos jours, comme on ne le sait que trop bien, les coulisses idéologiques, tout autant que la scène politique et la vie sociale, sont envahies par ce qu'on appelle poliment le «néo-libéralisme», et politiquement la «droite». Il est presque aussi évident (le récent revirement sur la scène politique française explique quelque peu l'hésitation) que la gauche, si présente hier encore, est tellement silencieuse qu'on pourrait bien croire que les foudres de la droite l'ont atterrée. Cet ouvrage, analysant avec discernement ce mouvement, sous une perspective de "gauche," est donc bien rafraîchissant.

Comme on peut bien s'y attendre dans un recueil de textes de ce genre, toutes les contributions ne sont pas du même calibre, et certaines pourraient décevoir. On trouve parfois des longueurs et des répétitions. L'idée, par exemple, que le néo-libéralisme n'est qu'un retour éhonté à Adam Smith est souvent reprise, comme si le manque d'originalité des penseurs néo-libéraux constituait leur principal vice. On trouve également quelques coquilles surtout dans les citations en anglais ( $j$ 'en ai noté trois, par exemple, dans celle de la page 187). Mais ce sont là des péchés presque mignons car les percées théoriques incisives sont fréquentes. Je ne les énumérerai pas toutes mais essaierai plutôt d'en donner un aperçu. Au préalable, cependant, il est indispensable de remercier Lizette Jalbert et Lucille Beaudry à qui l'on doit ce très utile instrument de compréhension et d'analyse d'un phénomène marquant de notre temps. De plus, leur excellente introduction situe bien les enjeux du débat en retraçant succinctement et précisément les facteurs historiques, politiques et théoriques qui peuvent ex- 
pliquer qu'aujourd'hui, comme elles le disent si bien, «la modernité a la figure du libéralisme.» (p. 16)

Cette introduction donne d'ailleurs le ton à l'ensemble de l'ouvrage qui est structuré autour de cette triple perspective historique, politique, et théorique. Les deux premiers chapitres offrent une vue d'ensemble historique et théorique de l'évolution du libéralisme. Le troisième offre également une perspective globale mais en plaçant l'accent, cette fois, sur les ramifications politiques du phénomène. Les auteurs de ce dernier chapitre nous rappellent, avec justesse, que le silence de la gauche nous semble d'autant plus lourd que notre horizon politique historique se limite trop souvent «au temps exaltant du radicalisme des années 1960." (p. 87) Le reste des contributions s'attaque à l'analyse de divers aspects théoriques de la problématique. On y trouve une critique du courant «institutionaliste», une comparaison des «néoAutrichiens» aux «néo-classiques», une critique des analyses de la gauche, une discussion de la "crise de la gouvernabilité" et, enfin, en conclusion, une évaluation de l'originalité du néolibéralisme.

Les premiers chapitres rendent compte de la résurgence du libéralisme classique. La contribution de François Houle a le grand mérite de mettre en relief les convergences et les divergences qui traversent le néo-libéralisme, et notamment en ce qui a trait aux diverses conceptions de l'État. On a tendance à oublier, et il est heureux que cet article nous le rappelle, que les conservateurs classiques, comme Burke par exemple, reconnaissent à l'État un rôle éthique important, alors que les néo-libéraux lui refusent "tout caracrère d'universel pour ne privilégier que le privé.» (p. 51) Ceci permet de mieux cerner le rapport entre ce retour au néo-libéralisme et l'enracinement de l'individualisme dans le tissu social. Comme le dit l'auteur, il est bien probable que, de plus en plus, cet individualisme occupe «une place plus grande au détriment de la justice et de l'égalité sociale.» (p. 59) 
C'est un peu à la même conclusion qu'arrive Dorval Brunelle qui veut également évaluer la force du néo-libéralisme en réexaminant la question de la place de l'État. Partant d'une présentation critique de Hayek, et de d'autres auteurs du même courant, il met en relief, dans un beau style limpide et précis, «leur refus de comprendre et de saisir les causes sociologiques et historiques qui ont prédéterminé la croissance de l'étatisme» (p. 79). Effectivement, on oublie bien vite que si Keynes, et d'autres, ont prôné l'étatisme ceci ne peut s'expliquer qu'en situant l'ensemble du processus dans son contexte social. Ce n'est certainement pas dû à «un cumul d'erreurs lié à un surcroît de théorisation.» L'identification de ce réductionisme méthodologique est l'axe autour duquel est élaborée une analyse incisive qui débouche sur la conclusion que si nous devons en partie à la droite la critique, bien valable, de l'étatisme ceci ne doit pas nous empêcher de voir les dissonances de cette thèse et ses effets pervers sur des valeurs de première importance pour une critique efficace des pouvoirs institués. Ceci conduit l'auteur à suggérer que c'est «la thèse de l'égalité qui a été le plus dénaturée dans la foulée de l'universalisation de l'étatisme et celle qu'il est impérieux de sauver à la fois contre l'État et contre le libéralisme. » (p. 81)

Les autres chapitres du livre s'attaquent à des problèmes plus circonscrits. De l'ensemble de ces contributions, deux méritent une mention spéciale. L'article de Maurice Lagueux, sur «Le néolibéralisme et la gauche», est d'un grand intérêt. Après avoir brièvement rappelé le préjugé favorable dont jouissait la gauche jusqu'à tout récemment, il présente une typologie succincte et précise des catégories d'arguments dirigés contre le néo-libéralisme. Axant son analyse autour du rapport entre science et idéologie, il évalue ensuite la pertinence et l'efficacité des réactions de la gauche. Avec grande dextérité, l'auteur parvient ainsi à montrer la complémentarité de ces deux formes de discours. Mieux encore, il suggère que ce sont les discours qui exercent une certaine 
autorité qui sont le plus aptes à fonctionner comme idéologie. Le discours scientifique jouissant d'un grand prestige à notre époque, il est donc parfaitement compréhensible qu'il puisse constituer un enjeu idéologique de choix.

L'auteur jette ainsi un éclairage saisissant sur la valeur scientifique et le potentiel idéologique des sciences sociales. Il devient possible d'évaluer à leur juste valeur les arguments, les doctrines, et les ouvres scientifiques de la «droite» tout en évitant les pièges idéologiques qui y sont recellés. Plusieurs exemples d'analyses scientifiques valables d'économistes néo-libéraux ainsi que leurs retombées idéologiques sont d'ailleurs présentés. Maurice Lagueux nous montre donc, concrètement et conceptuellement, comment une analyse de "gauche» accroît sa force en reconnaissant la force de celle de son adversaire. Comme il le dit lui-même, «il devient possible de faire la place qui leur revient, par exemple, aux analyses «scientifiques» du marché, tout en dénonçant l'idéologie qui y prend racine.» (p. 181) C'est un article à ne pas manquer.

Une autre contribution, bien intéressante en dépit de quelques lourdeurs stylistiques y compris l'utilisation, quelque peu agaçante, de la première personne du pluriel, est celle de Jacques Peltier. Partant, comme Lagueux, du rapport entre science et idéologie, l'auteur veut comparer l'école néo-autrichienne à l'école néoclassique, dont Hayek et Friedman font respectivement figures de proue. Plus spécifiquement, il veut montrer que «les choix entre les programmes de recherche ne sont explicables rationnellement qu'en incluant la problématique de la sauvegarde d'une philosophie politique.» (p. 141)

Bien que dans un bref article une telle thèse ne puisse être démontrée, Peltier éclaire brillamment deux dimensions fondamentales du rapport entre science et idéologie. Il dévoile, tout d'abord, les liens, à première vue cachés, entre les principes méthodologiques d'un programme de recherche, ses présupposés philosophiques et ses manifestations sociales. Il parvient ainsi à 
déceler l'axe de convergence des deux écoles néo-libérales qu'il situe au niveau de leur philosophie politique. Il montre, dans un deuxième temps, que les divergences les plus profondes entre ces deux écoles se situent au niveau épistémologique. Les néo-autrichiens accordent une place importante au subjectivisme mais rejettent, par contre, le «scientisme» ainsi que les méthodes qui font appel aux modèles économétriques visant à la prédiction. D'autre part, le paradigme méthodologique néo-classique est basé sur le postulat de l'objectivité des faits et se prête donc parfaitement à l'élaboration de modèles économétriques «prédisant» les effets des processus économiques. On peut ainsi mettre le doigt sur une ambiguité et une divergence, bien intéressante au sein des deux courants néo-libéraux concernant leur conception du rapport entre la science et la place de l'État. Bien que pour des raisons de philosophie politique les deux courants préconisent une intervention minimale de l'État, le «social-engineering», et donc à la limite la planification, est tout à fait compatible avec les thèses néo-classiques. Par contre, une telle conception du rôle de l'État est totalement incompatible avec l'individualisme méthodologique et le subjectivisme de l'école néo-autrichienne. Jacques Peltier nous donne ainsi accès aux structures fondamentales des perspectives néo-libérales. De ce fait, il jette un éclairage puissant sur les divergences et les convergences entre les deux écoles principales qui le constituent tout en montrant comment un programme de recherche véhicule nécessairement des valeurs sociales et idéologiques. C'est un autre article à ne pas manquer.

Dans une brève recension, surtout lorsqu'il s'agit d'un recueil de textes, il est bien difficile de rendre justice à tous les contributeurs, on ne peut que donner un avant-goût de ce que le lecteur trouvera dans l'ouvrage. Dans l'atmosphère actuelle de grand pragmatisme et de mise en veilleuse des projets de société quelque peu motivants, la parution de cet ouvrage pourrait surprendre. Mais je suis sûr que c'est un événement qui plaira beaucoup à ceux qui reconnaissent 
la valeur de la pensée critique. En effet, les fondements scientifiques et idéologiques des politiques actuelles sont enfin soumis à une critique sérieuse. Ce livre peut donc être perçu comme un vigoureux premier pas dans la voie tracée par Maurice Lagueux au sixième chapitre lorsqu'il émet le souhait que les intellectuels de gauche cessent de «se payer le luxe de n'opposer à la progression fulgurante de l'idéologie néo-libérale que des prises de position mécaniques et stériles que ces conceptions leur inspirent!» (p. 188) Il reste à souhaiter que les imitateurs seront nombreux.

Max Nemni

Université Laval 